\title{
Study on the Application of Gatifloxacin-A Novel Fluorescent System
}

\author{
Yuguang Lv ${ }^{1, \text { a }}$, Qi Shi ${ }^{1}$, Chunhui Shi ${ }^{2, b}$, Yun Zai ${ }^{1}$,Yushan Qin ${ }^{1}$ \\ ${ }^{1}$ College of Pharmacy, Jiamusi University, Jiamusi, Heilongjiang, 154007, China \\ ${ }^{2}$ First affiliated hospital of Jiamusi University, Jiamusi, Heilongjiang, 154002, China \\ a yuguanglv@163.com, b shichunhui66@163.com
}

Keywords: Gatifloxacin, Fluorescence, Applied research.

Abstract. we study the use of gatifloxacin with a novel tricyano-dihydrofuran fluorescent system. Gatifloxacin is a 8-methoxy-fluoroquinolone racemate which, in addition to having a broad spectrum of Gram-negative activity in addition to the activity of positive microorganisms, this product has antibacterial effect, bacterial DNA repair, transcription and replication can be suppressed. Such drugs are widely used at present, so they are getting more and more attention. Therefore, it is very important for these drugs to be tested efficiently. The application of the new type of tricyano-dihydrofuran fluorescence system can detect it efficiently, then we carry out a detailed application of its research.

\section{Introduction}

Quinolones, which target bacterial DNA, block DNA gyrase, can cause irreversible damage to bacterial DNA and are effective against bacteria. Quinolone antibiotics can be applied to the human body and can also be applied to animals. The antibacterial agent has the characteristics of wide antibacterial spectrum and strong activity, little side effect, no cross-resistance with other drugs, and has been widely used in animal husbandry and aquaculture Application, can be used for prevention and treatment of diseases ${ }^{1-5}$.There is an antibacterial drug called gatifloxacin, is a quinolone drugs, as humans gradually found that quinolones antibacterial effect is particularly good, so with quinolones synthesized more and more new structures, so for quinolones University drug testing methods are more and more important ${ }^{6-9}$.

\section{Experimental Methods.}

solution configuration, Preparation of new tricyne dihydrofuran standard solution, after the new tricyanohydrofuran synthesized has been vacuum-dried, $35.8 \mathrm{mg}$ of novel tricyano-dihydrofuran was accurately weighed and dissolved in an appropriate amount of chloroform. Fully dissolved, formulated as $1.5 \times 10^{-4} \mathrm{~mol} / \mathrm{L}$ of the mother solution, spare. Preparation of gatifloxacin (GFLX) standard solution, precision weighing $5 \mathrm{mg}$ of dry gatifloxacin (AR $\geq 99.0 \%$ ) standard, with a certain amount of methanol to be completely dissolved, dissolved, formulated to a concentration of $100 \mathrm{mg} / \mathrm{L}$ of the mother solution for use in the experiment can be Dilute the stock to the desired concentration. 


\section{Results and discussion}

\section{Fluorescence excitation and emission spectra}

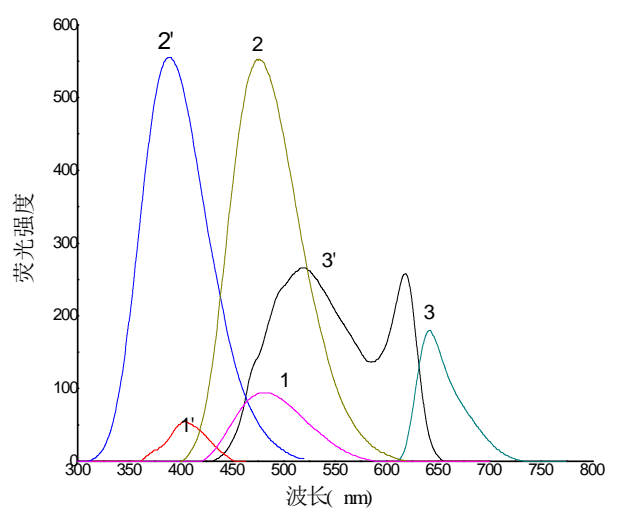

Fig. 1 Fluorescence excitation and emission spectra

1 gatifloxacin emits light 1 'gatifloxacin excitation light; 2 gatifloxacin - a novel tricyclic dihydrofuran emission light, 2' gatifloxacin - a novel tricyclic dihydrofuran excitation light; 3 Novel tricyano-dihydrofuran emission, 3 'new tricyclic dihydrofuran excitation

Take $3 \mathrm{ml}$ of new tricyano-dihydrofuran-gatifloxacin charge transfer complexes into the fluorescence excitation and emission spectra, and then separate the gatifloxacin working solution $3 \mathrm{ml}$ and new tricyanoid mother liquor $3 \mathrm{ml}$ under the same conditions of excitation and emission spectra for control experiments. The results shown in Figure 1, the new tricyano-dihydrofuran solution and gatifloxacin solution monomer has fluorescence characteristics. When the novel tricyano -dihydrofuran complexed with gatifloxacin, a charge-transfer complex was formed. The characteristic peak of the complex did not shift from the gatifloxacin monomer in their excitation and emission spectra The fluorescence intensity of the complex was significantly enhanced, indicating that the novel tricyano-dihydrofuran and gatifloxacin have formed charge-transfer complexes.

pH effect, Take seven dried plugged test tubes were added $0.5 \mathrm{ml}$ of new tricyclic dihydrofuran mother liquor and $4 \mathrm{ml}$ gatifloxacin working solution, a slight concussion to complete fusion, adding methanol was fixed, the test tube was kept at $40^{\circ} \mathrm{C}$ constant temperature Heat the water bath for 30 minutes, control the $\mathrm{pH}$ value within the range of $4.5 \sim 7.5$, remove it and place it to room temperature. The appropriate amount of 7 mixed solution were scanned at $354 \mathrm{~nm}$ fluorescence intensity. It can be seen from the figure that when the $\mathrm{pH}$ value of the system is controlled within the range of 4.5-5.0, the fluorescence intensity of the system continues to increase. When the $\mathrm{pH}$ value is 5.5 , the fluorescence intensity reaches the strongest. The peracid will destroy the complex structure. Therefore, $\mathrm{pH}=5.5$ is the best $\mathrm{pH}$ value.

Effect of DCDHF-2-V concentration, Take seven dried plugged test tube, were added $4 \mathrm{ml}$ of gatifloxacin and $0.5 \mathrm{ml}$ of new tricyclic dihydrofuran mother liquor, a slight concussion to complete fusion, adding anhydrous methanol volume, the tube was placed in a constant temperature water bath The concentration of DCDHF-2-V was determined by heating at room temperature, fixing the concentration of gatifloxacin, keeping the temperature at $40{ }^{\circ} \mathrm{C}$ in water bath for $30 \mathrm{~min}$ and $\mathrm{pH}=5.5$. The fluorescence intensity of the system at different concentrations of DCDHF-2-V was measured. $1.0 \times 10^{-3} \mathrm{~mol} . \mathrm{L}^{-1}-3.5 \times 10^{-4} \mathrm{~mol} . \mathrm{L}^{-1}$ system has a strong fluorescence intensity, continue to study.

Gatifloxacin complex for the best screening conditions, Amount of DCDHF-2-V affected, $0.1-0.7 \mathrm{ml}$ DCDHF-2-V with gradient of $0.1 \mathrm{ml}$ and $1.0 \mathrm{ml}$ and $2.0 \mathrm{ml}$ was added to the dried plugged test tube with $4 \mathrm{ml}$ of gatifloxacin respectively, and the volume was fixed with anhydrous methanol, In $40{ }^{\circ} \mathrm{C}$ water bath for $30 \mathrm{~min}$, placed at room temperature, measured fluorescence intensity The measured results show that when the amount of DCDHF-2-V $0.5 \mathrm{~mL}$ fluorescence enhancement. Low concentrations and high concentrations result in lower fluorescence (low concentration, incomplete reaction; high concentration, low fluorescence). 
The amount of gatifloxacin affected, Take 1-8 ml, gradient of $1 \mathrm{~g}$ of gatifloxacin solution, were added to $0.5 \mathrm{mLDCDHF}-2-\mathrm{V}$ with a plugged dried test tube, add anhydrous methanol volume, shake well, $40^{\circ} \mathrm{C}$ water bath temperature $30 \mathrm{~min}$, cooled to room temperature, measured fluorescence intensity The measured results show that when the dosage of gatifloxacin is controlled at $4 \mathrm{ml}$, the fluorescence intensity of the charged complex is the highest, which is the best condition at this time.

\section{Temperature and time effects}

Temperature effects, According to the above experimental method, fixed the other experimental conditions remain unchanged, the temperature of the water bath were controlled at $20-60^{\circ} \mathrm{C}$, with a plug containing the test tube was heated for 30 minutes, removed and placed to room temperature. The fluorescence intensity of the complex was measured as shown in Figure 2. The data show that the temperature increase helps to increase the fluorescence intensity of the system, but the complex tends to be stable when the temperature reaches $40^{\circ} \mathrm{C}$ (warming facilitates the formation of the complex, but the temperature Should not be too high, damage components), so the optimal reaction temperature of the system is $40^{\circ} \mathrm{C}$.

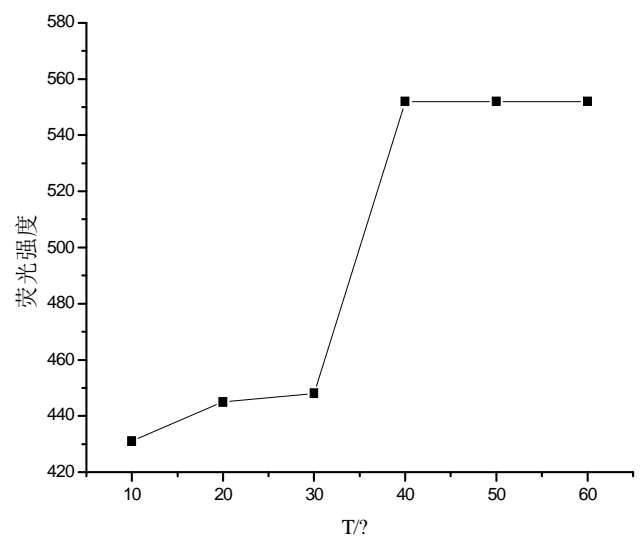

Fig. 2 Temperature effects

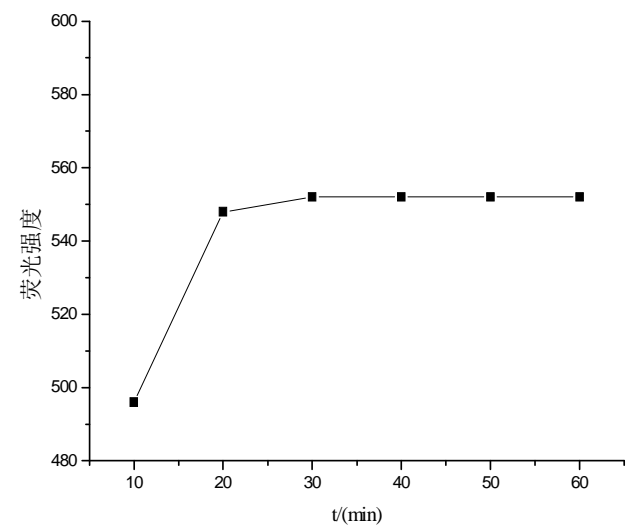

Fig. 3 The impact of time

The impact of time, Fixing the other experimental conditions (single variable method), under the conditions of temperature determination, respectively, in the reaction 10min 60min selected six full time period when detecting the fluorescence intensity of the complex, the results shown in Figure 3 , Gatifloxacin andThe new tricyano-dihydrofuran reacted completely at 30 minutes.

Reaction mechanism, Novel tricyano-dihydrofuran has a planar molecular structure (D- $\pi$-A type), is a strong electron acceptor material. Gatifloxacin C-7 position even with a lone pair of nitrogen atoms, and the new electron acceptor of a new type of cyanide dihydrofuran under the appropriate conditions to occur in charge-transfer reaction, a new charge-transfer complex Things. The reaction principle is as follows: 


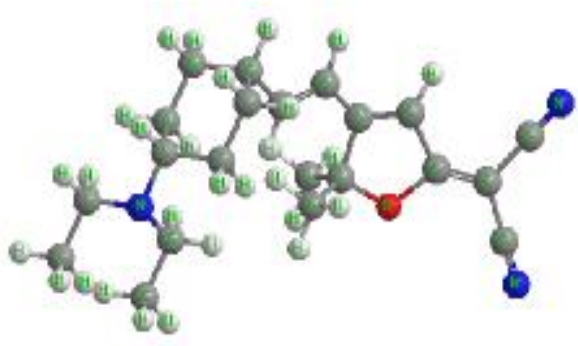

DCDHF-2-V

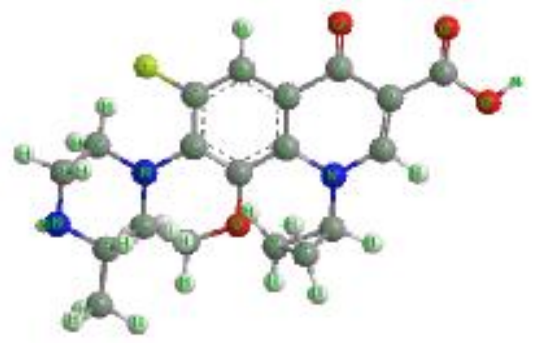

Gatifloxacin

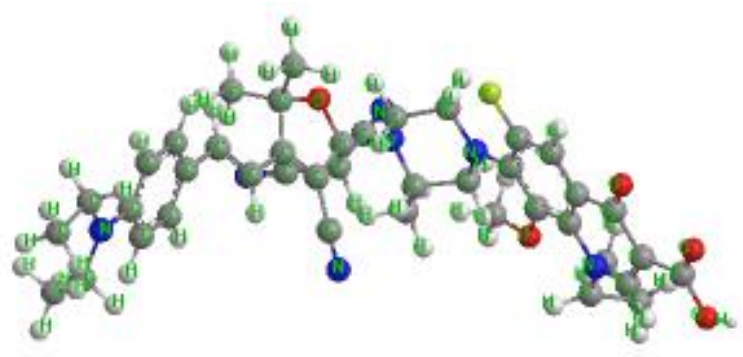

Complex

Fig. 4 Reaction mechanism

Sample Determination, 5 tablets of gatifloxacin in a certain area , mortar into powder, accurately weighed total amount of grinding $1 / 5$ (equivalent to $100 \mathrm{mg}$ drugs), with an appropriate amount of anhydrous methanol to dissolve, microwave ultrasound or glass rod Soluble. To be completely dissolved, anhydrous methanol volume $(100 \mathrm{ml})$. Pipette exactly $10 \mathrm{~mL}$ sample solution, diluted to $1 \times 10^{-3} \mathrm{~mol} / \mathrm{L}$ anhydrous methanol, spare. Using a series of optimization conditions explored by the above experimental scheme, the content of norfloxacin in the sample was determined, and six parallel determination results were selected. The results are shown in Table1Experimental data. In order to increase the effectiveness of the results, standard addition method was used to determine the recovery rate of the drug.

Table1. sample measurement results and recovery rate

\begin{tabular}{ccccc}
\hline sample & Add amount (mg) & Measured value (mg) & Recovery rate (\%) & RSD (\%) \\
\hline 1 & 9.53 & 9.55 & 100.21 & 1.07 \\
2 & 9.53 & 9.61 & 100.83 & 1.13 \\
\hline
\end{tabular}

\section{Conclusions}

Gatifloxacin In the chloroform and methanol solution system, gatifloxacin can form a stable charge-transfer complex with the novel tricyano-dihydrofuran. The optimum $\mathrm{pH}$ value of this system is 5.5 , the reaction temperature is $40{ }^{\circ} \mathrm{C}$ and the reaction time is $30 \mathrm{~min}$. The fluorescence intensity of the system is significantly higher than ofloxacin. Therefore, this method can be used as a new method for the determination of ofloxacin in medicine, and the reaction conditions are mild, high speed and high sensitivity.

\section{Acknowledgements}

This work was financially supported by the National Science Foundation of China (No. 21346006), Department of scientific research project in Heilongjiang province (No. B2017015), Scientific research project of Heilongjiang province education department (No.12541783), National 
project training project of Jiamusi University (No.JMSUJCGP 2016-003), Scientific research project of Jiamusi University (JMSUJCMS2016-013).

\section{References}

[1] Hui Wang,Zhikuan Lu,Samuel.J.Modifications of DCDHF single molecule fluorophores to impart water solubility[J].Tetrahedron Letters,2007,48: 3471-3474.

[2] EnriqueFont-Sanchis,,a Raquel E. Galian,b Francisco J.Alkoxy-styryl DCDHF fluorophores[J]. Physical Chemistry Chemical Physics ,2010, 12:768-7771.

[3] Wissam Bentoumi.Concise Multigram-Scale Synthesis of Push-Pull Tricyanofuran-Based Hemicyanines with Giant Second-Order Nonlinearity: An Alternative for Electro-optic Materials [J]. Chem. Soc.Rev,2014, 20: 8909-8913.

[4] Hui Wang, Zhikuan Lu,.The influence of tetrahydroquinoline rings in dicyanomethylene dihydrofuran (DCDHF) single-molecule fluorophores [J].Tetrahedron, 2006, 10: 103-114.

[5] Mingqian He, Thomas M. Leslie, John A. Sinicropi. r-Hydroxy Ketone Precursors Leading to a Novel Class of Electro-optic Acceptors[J]. Chem. Mater.2002, 14: 2393-2400.

[6] Rurack.K., Genger,U. R. Rigidization, preorientation and electronic decoupling-the 'magic triangle' for the design of highly efficient fluorescent sensors and switches.[J]. Chem. Soc.Rev. 2002,31:116-127.

[7] Pearce,D.A.,Jotterand,N. Derivatives of 8-Hydroxy-2-methyl quinoline are powerful prototypes for zinc sensors in biological systems, [J]. J.Am. Chem.Soc, 2001, 123(21) 5160-5161.

[8] Sigmund.H.Pfleiderer.W. A new type of labeling of nucleosides and nucleotides, [J]. Helv Chim Acta,2003,86(7):2299-2334.

[9] Adamczyk.M.,Chan.C.,Fino.J. et al.,Synthesis of 5-and 6-hydroxymethyl fluoresce in phosphora inidites.[J]- J.Org.Chem.2000,65(2):596-601. 